The Journal of Bone E Joint Surgery
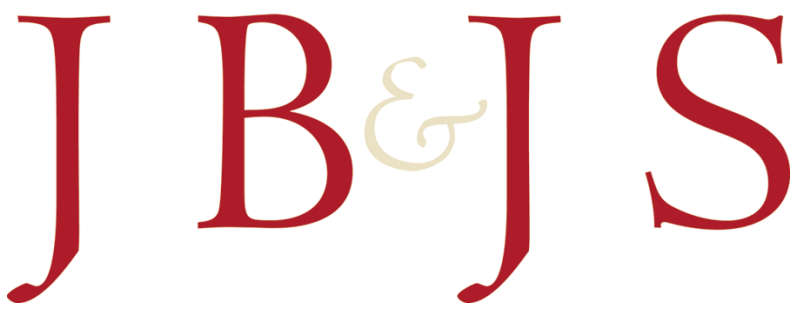

This is an enhanced PDF from The Journal of Bone and Joint Surgery

The PDF of the article you requested follows this cover page.

\title{
Ulnar Shortening Osteotomy in Idiopathic Ulnar Impaction Syndrome
}

Goo Hyun Baek, Moon Sang Chung, Young Ho Lee, Hyun Sik Gong, Sanglim Lee and Hyung Ho Kim J Bone Joint Surg Am. 2005;87:2649-2654. doi:10.2106/JBJS.D.02983

This information is current as of August 27, 2009

Supplementary material

Reprints and Permissions

Publisher Information
Commentary and Perspective, data tables, additional images, video clips and/or translated abstracts are available for this article. This information can be accessed at http://www.ejbjs.org/cgi/content/full/87/12/2649/DC1

Click here to order reprints or request permission to use material from this article, or locate the article citation on jbjs.org and click on the [Reprints and Permissions] link.

The Journal of Bone and Joint Surgery 20 Pickering Street, Needham, MA 02492-3157 www.jbjs.org 


\title{
Ulnar SHORTENING OSTEOTOMY IN IDIOPATHIC ULNAR IMPACTION SYNDROME
}

\author{
By Goo Hyun Baek, MD, Moon Sang Chung, MD, Young Ho Lee, MD, \\ Hyun SiK Gong, MD, SANGLim Lee, MD, and Hyung Ho Kim, MD \\ Investigation performed at the Department of Orthopaedic Surgery, Seoul National University College of Medicine, Seoul, South Korea
}

\begin{abstract}
Background: Idiopathic ulnar impaction syndrome can be defined as a degenerative condition of the ulnar aspect of the wrist in patients with congenital or dynamic positive ulnar variance without a history of fracture or premature physeal arrest. The purpose of this study was to evaluate the clinical features of idiopathic ulnar impaction syndrome and the outcomes of ulnar shortening osteotomy for this group of patients.
\end{abstract}

Methods: Thirty-one wrists in twenty-nine patients with idiopathic ulnar impaction syndrome were treated with an ulnar shortening osteotomy. Ulnar variance was measured on an anteroposterior radiograph of the wrist, and radioulnar distance was measured on a lateral radiograph, with the forearm in neutral rotation, to evaluate any displacement of the ulnar head from the distal aspect of the radius. All patients were followed clinically and radiographically for a mean of thirty-two months.

Results: An average preoperative ulnar variance of $+4.6 \mathrm{~mm}$ (range, 2 to $7.5 \mathrm{~mm}$ ) was reduced to an average of -0.7 $\mathrm{mm}$ (range, -4 to $+1 \mathrm{~mm}$ ) postoperatively. Preoperatively, the modified Gartland and Werley score was an average (and standard deviation) of $69.5 \pm 7.6$, with twenty-four wrists rated poor and seven rated fair. Postoperatively, the score improved to an average of $92.5 \pm 8.0$, with twenty-four wrists rated excellent; five, good; one, fair; and one, poor. Dorsal subluxation of the distal aspect of the ulna was found concomitantly in nine wrists, and it was found to be reduced by the shortening osteotomy. Seven patients had cystic changes in the carpal bones preoperatively, but these were not evident one to two years after the operation.

Conclusions: Ulnar shortening osteotomy improved wrist function in patients with idiopathic ulnar impaction syndrome and reduced the subluxation of the distal radioulnar joint, which is commonly found in these patients. Degenerative cystic changes of the ulnar carpal bones appear to resolve following the shortening osteotomy.

Level of Evidence: Therapeutic Level IV. See Instructions to Authors for a complete description of levels of evidence.

$\mathrm{U}$ lnar impaction syndrome, which is frequently seen in patients with positive ulnar variance, can be defined as a degenerative condition characterized by ulnar wrist pain, swelling, and limitation of motion related to excessive load-bearing across the ulnar aspect of the wrist'. The common acquired predisposing conditions for this syndrome include malunion of a distal radial fracture, premature physeal arrest of the distal part of the radius, and a previous radial head resection or an Essex-Lopresti injury ${ }^{1-4}$. However, in patients who have congenital or dynamic positive ulnar variance with wrist pronation and forceful grip without any history of fracture or premature physeal arrest, the condition is termed

A video supplement to this article will be available from the Video Journal of Orthopaedics. A video clip will be available at the JBJS web site, www.jbjs.org. The Video Journal of Orthopaedics can be contacted at (805) 962-3410, web site: www.vjortho.com. "idiopathic" ulnar impaction syndrome. The purpose of this study was to evaluate the clinical features and outcomes of idiopathic ulnar impaction syndrome in a group of patients treated surgically with an ulnar shortening osteotomy.

\section{Materials and Methods}

Demographic and Surgical Data

Thirty-one wrists in twenty-nine consecutive patients with

I idiopathic ulnar impaction syndrome were treated with an ulnar shortening osteotomy. There were seven men and twenty-two women with an average age of 40.5 years (range, sixteen to fifty-seven years). None of them had a history of fracture in the wrist or forearm.

The most common presenting symptom was ulnar wrist pain, and additional symptoms included a decreased range of motion, weakness of grip, and swelling or clicking of the wrist. The symptoms had a gradual onset and were related to the oc- 
THE JOURNAL OF BONE \& JOINT SURGERY • JBJS.ORG VOLUme 87-A · Number 12 - DECEMBER 2005
UlnaR SHortening OSTEOTOMY IN IDIOPATHiC UlnaR IMPACTION SYNDROME

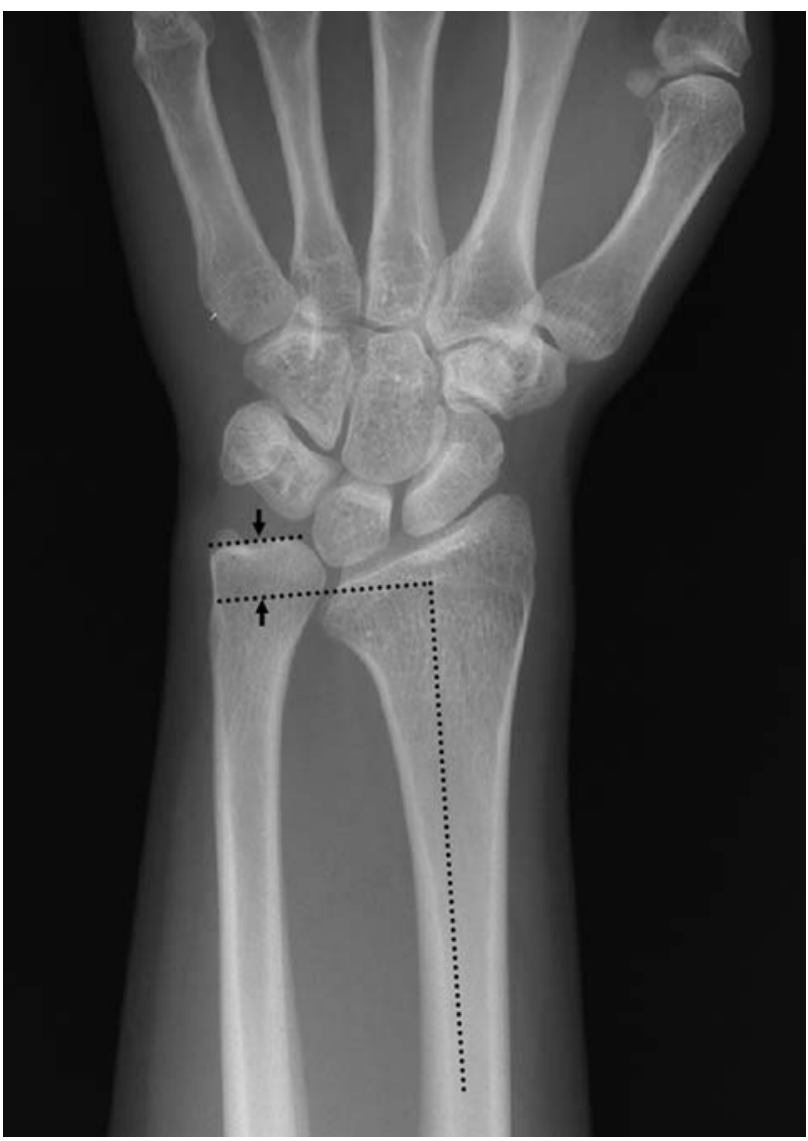

Fig. 1-A

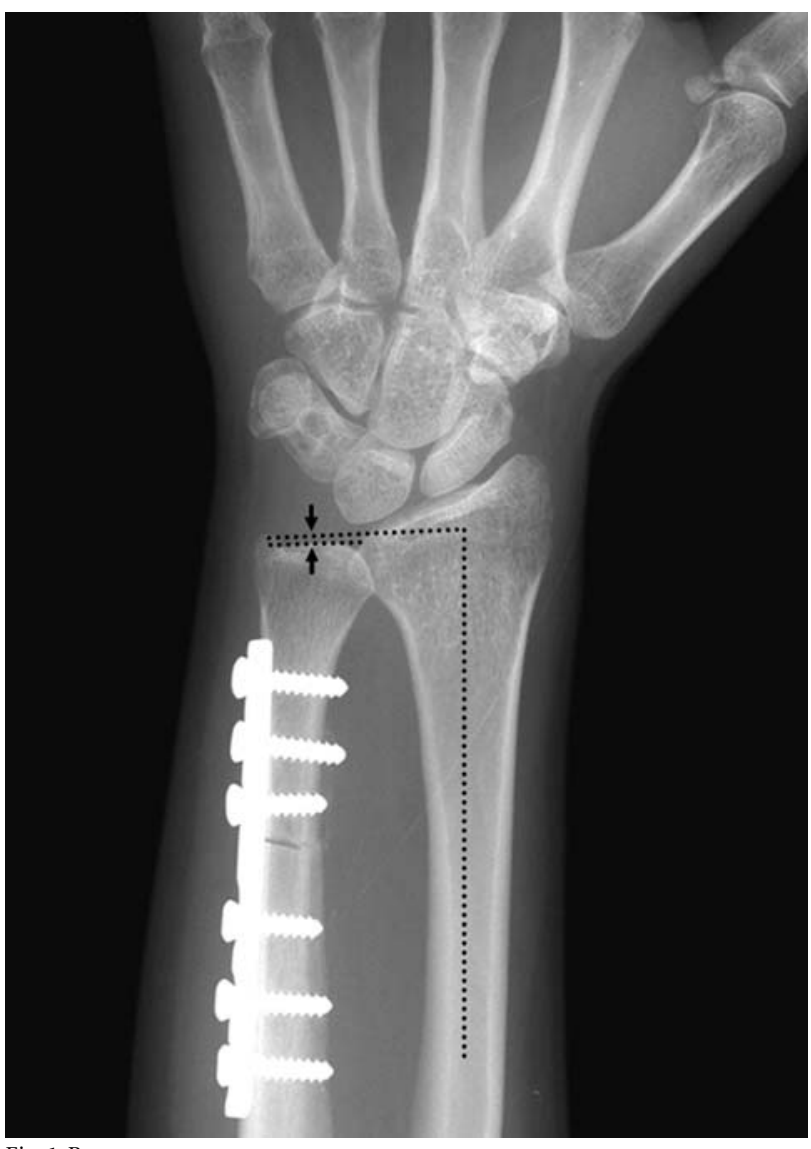

Fig. 1-B

Fig. 1-A Measurement of ulnar variance (arrows) on a preoperative anteroposterior radiograph. Fig. 1-B Measurement of ulnar variance (arrows) on an anteroposterior radiograph made after the ulnar shortening osteotomy and fixation with a dynamic compression plate and screws.

cupation and sports activities of the patients. Patients with definite traumatic injuries to the wrists were excluded from the study.

The diagnosis was made on the basis of a review of the history; the findings of the physical examination, including the use of a provocative maneuver, such as the ulnocarpal stress test ${ }^{1}$, in which the wrist is deviated ulnarward and the forearm is pronated; and plain radiographs that showed positive ulnar variance with or without cystic change(s) of the lunate or triquetrum. In twenty-five wrists, a magnetic resonance image was also acquired to identify abnormalities of the triangular fibrocartilage complex.

The inclusion criteria for the diagnosis were (1) no history of trauma of the forearm and wrist; (2) a history of ulnar wrist pain that was worsened by pronation and ulnar deviation of the wrist with such activities as opening a jar, squeezing a wet towel, typing, or changing the gearshift in an automobile; (3) a positive provocation test (ulnocarpal stress test); and (4) positive ulnar variance with or without cystic changes of the carpus on plain radiographs.

The exclusion criteria were radiographic evidence of an old fracture of the forearm or wrist or radiographic evidence of congenital anomalies such as a Madelung deformity.

Nonoperative treatment, including nonsteroidal anti- inflammatory medications and splinting, was tried for more than three months in all patients. In seventeen patients who showed cystic changes of the carpal bones on the radiographs or a degenerative tear of the triangular fibrocartilage complex on the magnetic resonance imaging scan, arthroscopy of the wrist was done along with the ulnar shortening procedure. The dominant hand was involved in seventeen patients.

The goal of the shortening osteotomy was to obtain a final ulnar variance of between 0 and $-1 \mathrm{~mm}$. All osteotomies were done transversely and were fixed with a six or seven-hole 3.5-mm standard compression plate (Stryker Trauma, Selzach, Switzerland) in twenty-four patients and with a one-third tubular plate (Stryker Trauma) in seven patients in whom the ulna was too small for a standard compression plate. Postoperatively, the patients were managed for the first two weeks with a long arm splint that was then converted to a removable long arm splint and was worn for an additional period of four to eight weeks.

\section{Radiographic Evaluation}

Perioperative ulnar variances were measured with the method of perpendiculars, whereby a line is drawn perpendicular to the longitudinal axis of the radius at its distal ulnar aspect, and the distance between it and the line at the end of the ulna is 
ThE JOURNAL OF BONE \& JOINT SURGERY • JBJS.ORG VOLUme 87-A · Number 12 - DeCEMber 2005

measured $^{2}$ (Figs. 1-A and 1-B). Special attention was given to problems of the distal radioulnar joint, and patients with localized pain in the distal radioulnar joint rather than on the ulnar side of the wrist were excluded from this study. However, patients who presented with ulnar impaction syndrome and coexistent problems of the distal radioulnar joint were included. To quantify the status of the distal radioulnar joint, the radioulnar distance $^{5}$ was measured perioperatively on a true lateral radiograph of the wrist, in which the pisoscaphoid distance was $<3$ $\mathrm{mm}$ (Figs. 2-A and 2-B). The anteroposterior radiograph of the wrist was made with the shoulder abducted to $90^{\circ}$, the elbow flexed to $90^{\circ}$, and the forearm in neutral rotation. The lateral radiograph was made with the shoulder at $0^{\circ}$ abduction and the elbow and forearm in the same amount of flexion and rotation.

\section{Follow-up}

The average duration of postoperative follow-up was thirty-two months (range, eighteen to sixty months). All twenty-nine patients had a preoperative evaluation of the wrist with use of the scoring system of Gartland and Werley as modified by Chun and Palmer ${ }^{3}$. The patients were seen postoperatively every two

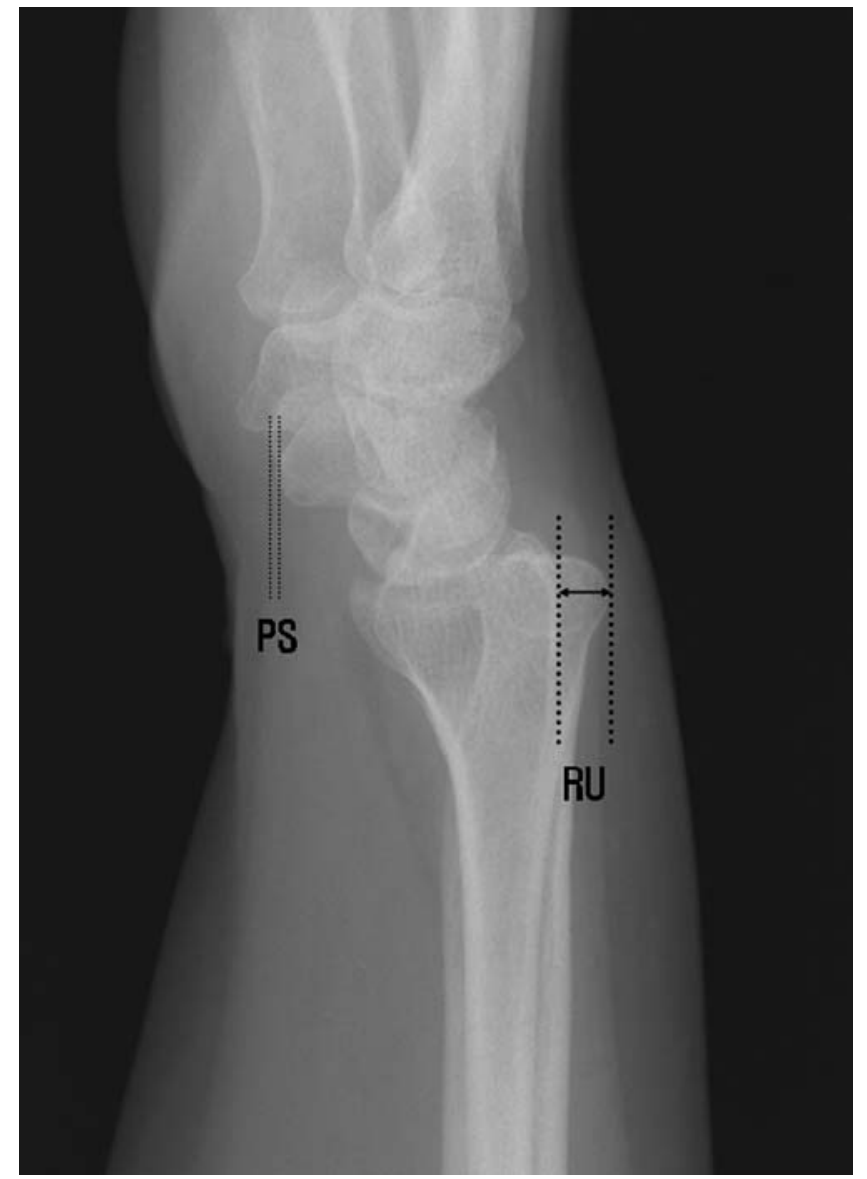

Fig. 2-A

Figs. 2-A and 2-B Radiographs of the wrist of a patient who had an ulnar shortening osteotomy. Fig. 2-A Preoperative lateral radiograph showing the measurement of the radioulnar distance (RU). The pisoscaphoid distance (PS) was used to define a true lateral radiograph. Fig. 2-B Postoperative radiograph showing a decrease in the radioulnar distance.
UlnaR Shortening OSTEOTOMY IN IDIOPATHIC ULNaR IMPACTION SYNDROME

to four weeks until radiographic union was confirmed, and they were examined with use of the evaluation system to obtain a wrist score at three and six months and once yearly thereafter.

\section{Statistical Analysis}

Perioperative wrist scores and ulnar variances were analyzed with use of the paired Student $t$ test. In patients with increased radioulnar distances, the changes in these distances and ranges of forearm motion after the ulnar shortening osteotomy were analyzed with the Wilcoxon signed-rank test. Correlation analysis was done to assess any relationship between the ulnar variance and the radioulnar distance. Statistical analysis was conducted with the SPSS software package (SPSS, Chicago, Illinois), and significance was established at $\mathrm{p}<0.05$.

\section{Results}

\section{Clinical Evaluations}

7 he preoperative wrist score was an average (and standard deviation) of $69.5 \pm 7.6$, with twenty-four wrists rated poor and seven rated fair. At the time of the final follow-up, the average score had improved to $92.5 \pm 8.0$, with twenty-four wrists

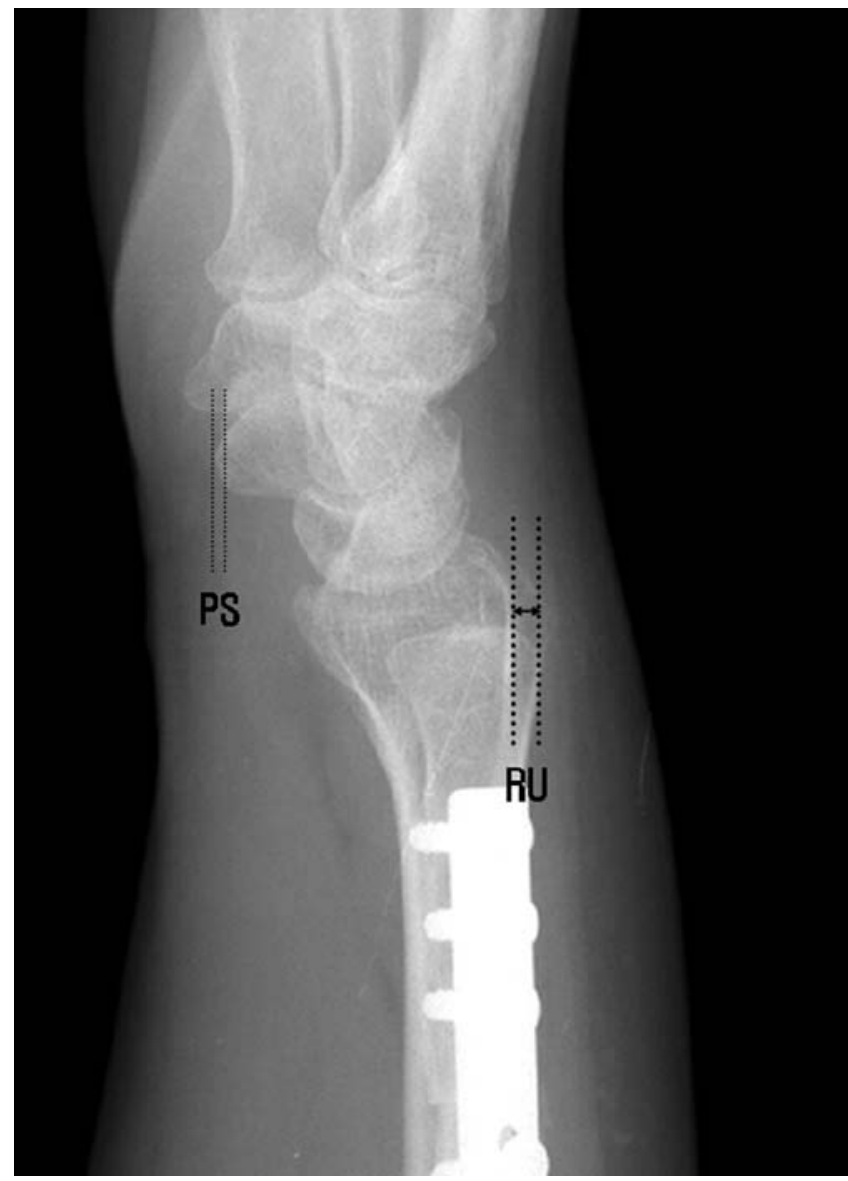

Fig. 2-B 
The Journal of Bone \& JOINT SURGERY • JBJS.ORG Volume 87-A · Number 12 - DeCEMber 2005
UlnaR Shortening OSTEOTOMY IN IDIOPATHIC UlnaR

IMPACTION SYNDROME

TABLE I Outcome According to Wrist Grading System of Chun and Palmer ${ }^{3}$

\begin{tabular}{|lrrr|}
\hline & Preoperative* & Postoperative* & P Value \\
\hline Wrist score & $69.5 \pm 7.6$ & $92.5 \pm 8.0$ & $<0.0001$ \\
Subjective pain score & $9.3 \pm 3.8$ & $17.6 \pm 3.1$ & $<0.0001$ \\
Subjective motion score & $6.9 \pm 2.1$ & $9.4 \pm 1.1$ & $<0.0001$ \\
Objective motion score & $7.8 \pm 0.9$ & $9.2 \pm 1.3$ & $<0.0001$ \\
Objective strength score & $7.6 \pm 1.9$ & $9.0 \pm 1.5$ & 0.0008 \\
\hline *The values are given as the mean and the standard deviation. & & \\
\hline
\end{tabular}

rated excellent; five, good; one, fair; and one, poor. Each component of the wrist score, including subjective pain and motion and objective motion and strength scores, also improved postoperatively (Table I). In two patients who had a bilateral osteotomy, the grip strength had improved $>20 \%$ postoperatively and was rated as excellent in objective strength.

Of the twenty-nine patients with idiopathic ulnar impaction syndrome, nine were diagnosed clinically as having concomitant problems of the distal radioulnar joint, with tenderness, limitation of forearm rotation, and apparent dorsal prominence of the distal aspect of the ulna. After ulnar shortening, these symptoms also improved. Tenderness of the distal radioulnar joint disappeared as did the dorsal prominence of the distal aspect of the ulna, as shown by the decreased radioulnar distance in the radiographic findings. Rotation of the forearm in the nine patients also improved from an average of $122^{\circ}$ (range, $90^{\circ}$ to $160^{\circ}$ ) to an average of $148^{\circ}$ (range, $140^{\circ}$ to $\left.160^{\circ}\right)$ postoperatively $(\mathrm{p}=0.018)$.

Union of the osteotomy was achieved at an average of 11.5 weeks (range, eight to sixteen weeks). There were no nonunions or infections. Two patients had development of a postoperative reflex sympathetic dystrophy, which improved nine months and eleven months, respectively, after the operation. However, the final wrist score was fair in one patient and poor in the other.

\section{Radiographic Findings}

The average ulnar variance was $+4.6 \mathrm{~mm}$ (range, 2 to $7.5 \mathrm{~mm}$ ) preoperatively and $-0.7 \mathrm{~mm}$ (range, -4 to $+1 \mathrm{~mm}$ ) postoperatively $(\mathrm{p}<0.0001)$. In the nine patients with clinical dorsal subluxation of the distal aspect of the ulna, the preoperative radioulnar distance averaged $+7.1 \mathrm{~mm}$ (range, +4.5 to +12 $\mathrm{mm}$ ) on the true lateral radiograph. This distance decreased to an average of $+2.8 \mathrm{~mm}$ (range, +2 to $+4 \mathrm{~mm}$ ) postoperatively $(\mathrm{p}=0.008)$. A positive correlation was detected between ulnar variance and the radioulnar distance, and the coefficient of correlation was $0.376(\mathrm{p}=0.037)$.

Preoperatively, seven of the thirty-one wrists showed cystic changes in the carpal bones on plain radiographs; four had changes in the lunate; two, in both the lunate and the triquetrum; and one, in the triquetrum. One to two years after the ulnar shortening osteotomy, most of these cystic changes were absent from the radiographs (Figs. 3-A and 3-B).

\section{Arthroscopic Findings}

Arthroscopy of the wrist joint was done in addition to an ulnar shortening osteotomy in seventeen patients who had cystic changes of the carpus on the radiographs or evidence of a degenerative tear of the triangular fibrocartilage complex on magnetic resonance imaging. Degeneration of the triangular fibrocartilage complex was seen in all seventeen patients, and a central perforation was noted in five patients. All patients had chondromalacia ${ }^{6}$ of the lunate. Grade-I chondromalacia (localized softening of the articular cartilage with minimal or no break on the surface) was found in three patients; grade II (an area of fibrillation or fissuring of articular cartilage with an irregular surface), in five; grade III (definite fibrillation with fissuring of articular cartilage extending down to the subchondral bone), in eight; and grade IV (exposed and eroded subchondral bone), in one patient. The lunotriquetral ligament was found to have a partial tear and minimal laxity in nine patients. Seven patients had chondromalacia in the triquetrum, with grade I in three, grade II in three, and grade III in one. Arthroscopic débridement of the triangular fibrocartilage complex, lunate, lunotriquetral ligament, and/or triquetrum was performed in these patients.

In three patients who had cystic changes in the lunate and chondromalacia that was classified as grade III or higher, a subchondral microfracture of the lunate was created. With the arthroscope in the 3-4 portal and the working instrument in the $6 \mathrm{R}$ portal, we exposed the crater after lifting and débriding a cartilage flap, if present. Then we cleared the crater and, with a banana knife (Linvatec, Largo, Florida), fractured the subchondral bone down to bleeding cancellous bone.

\section{Discussion}

T lnar variance in Asians is usually positive, with a mean

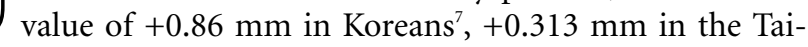
wanese $^{8}$, and $+0.20 \mathrm{~mm}$ in the Japanese population ${ }^{9}$, whereas ulnar variance in the white population is usually negative, with a mean value of $-0.9 \mathrm{~mm}$ in Americans ${ }^{10}$ and $-0.84 \mathrm{~mm}$ in Danes ${ }^{11}$. These findings suggest that the prevalence of idiopathic ulnar impaction syndrome may be more common in Asians than in white individuals.

Nakamura et al. ${ }^{5}$ reported that, in normal wrists, the difference between the radioulnar distance in the right and left wrists does not exceed $4 \mathrm{~mm}$ when the difference in the piso- 


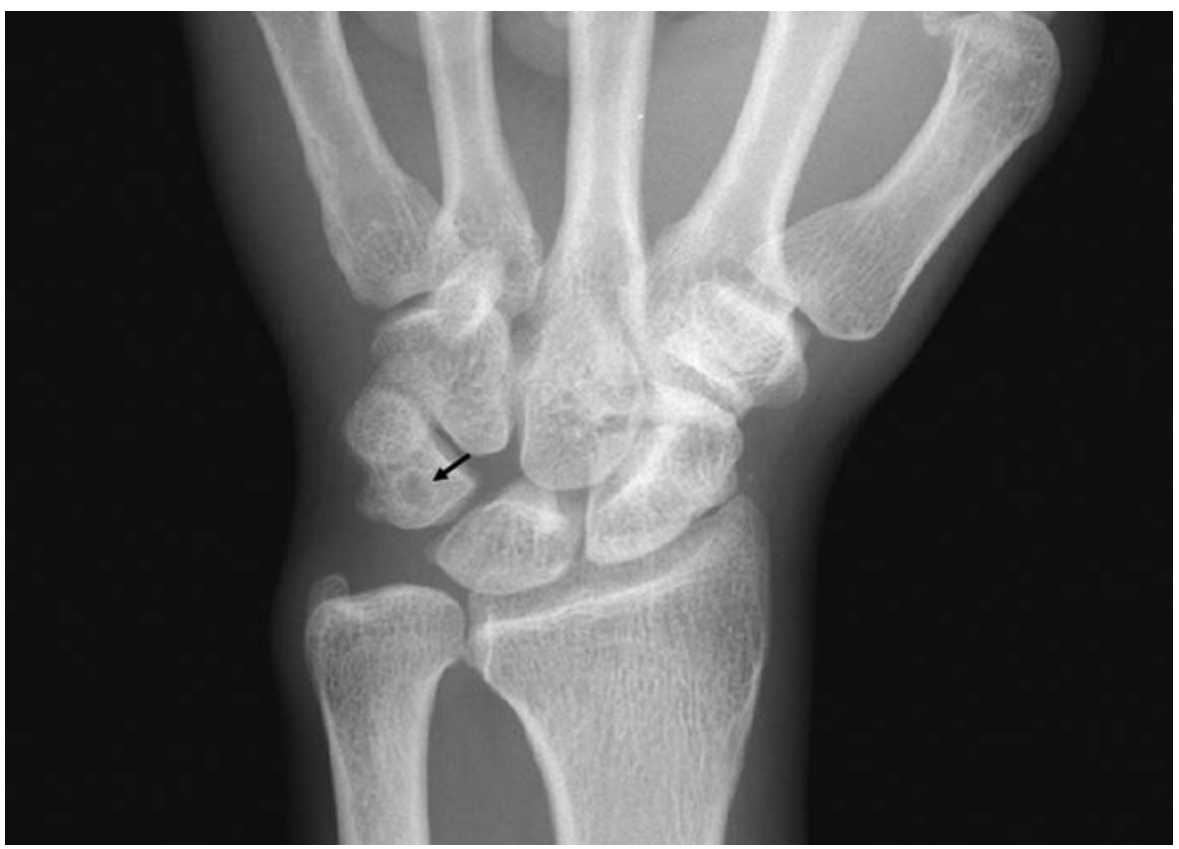

Fig. 3-A

Figs. 3-A and 3-B A patient with cystic changes. Fig. 3-A Preoperative anteroposterior radiograph showing a triquetral cyst (arrow).

scaphoid distance is $<3 \mathrm{~mm}$. With use of this finding as the criterion for a true lateral radiograph, the radioulnar distance was measured in patients who presented clinically with dorsal subluxation of the distal aspect of the ulna and was found to have decreased postoperatively. With ulnar shortening, the extrinsic ulnocarpal ligaments are tightened, perhaps stabilizing the distal aspect of the ulna and the lunotriquetral articulation $^{1,12}$. Another possible explanation is that the ulnar aspect of the carpus may be contacting the very long ulna and driving it out dorsally. When the ulna is shortened, the impaction stops and the distal aspect of the ulna can settle back into the sigmoid notch.

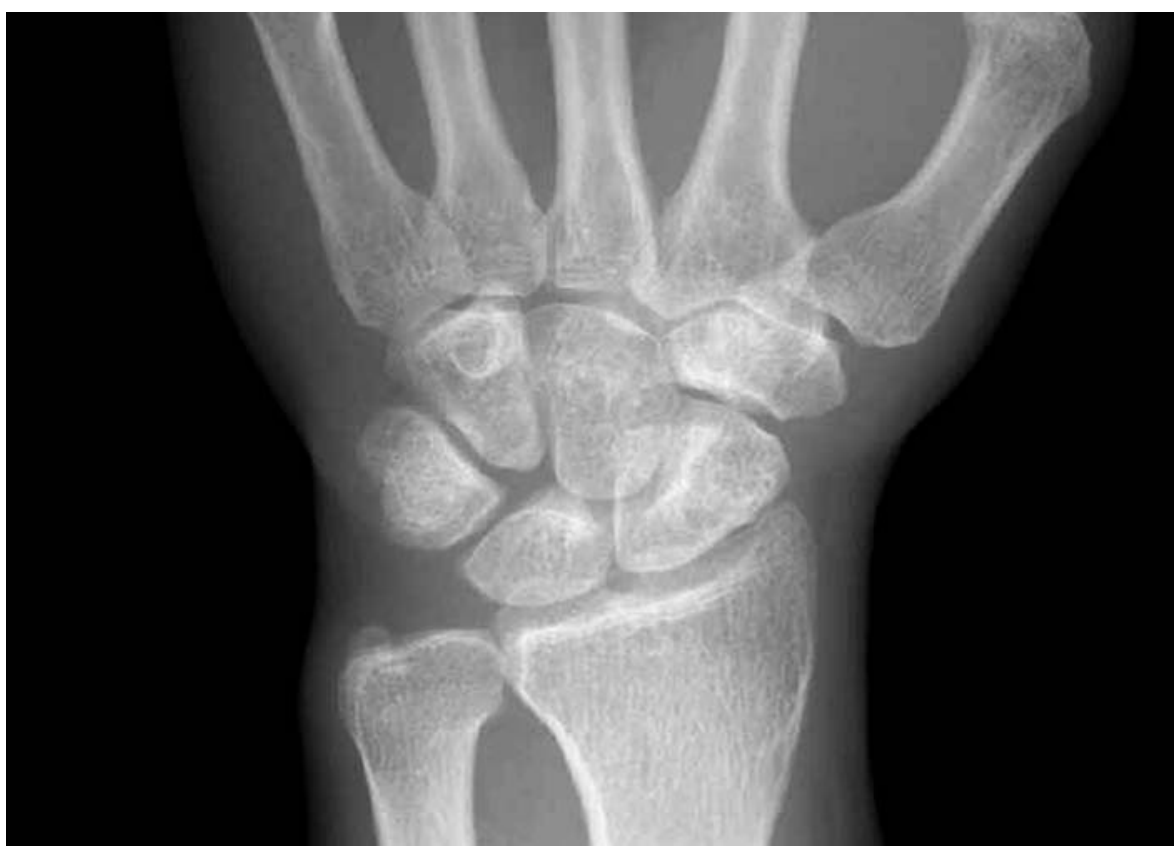

Fig. 3-B

A radiograph made twelve months after the ulnar shortening osteotomy showing that the cyst is no longer apparent. 
THE JOURNAL OF BONE \& JOINT SURGERY • JBJS.ORG Volume 87-A · Number 12 - DeCember 2005
ULNAR SHORTENING OSTEOTOMY IN IDIOPATHIC ULNAR IMPACTION SYNDROME
This advantage of the ulnar shortening osteotomy may explain the postoperative radiographic and clinical improvement in patients with idiopathic ulnar impaction syndrome. The patients with problems at the distal radioulnar joint in the present study showed no important degenerative arthritic changes in that joint. While some authors have stated that ulnar shortening osteotomy is contraindicated in patients with instability or degenerative arthritis of the distal radioulnar joint because of the risk of injuring the already compromised joint ${ }^{13}$, the findings of the present study suggest that the osteotomy can be beneficial in patients with coexistent distal ulnar subluxation, as long as the distal radioulnar joint does not have extensive arthritic changes.

The present study also found a positive correlation between ulnar variance and the radioulnar distance, which suggests that a wrist with a greater amount of ulnar variance has a greater likelihood of subluxation of the distal radioulnar joint. The findings in our study population suggest that, when the positive ulnar variance is $>4.5 \mathrm{~mm}$, subluxation of the distal radioulnar joint is a commonly associated finding in patients with idiopathic ulnar impaction syndrome.

Seven patients had cystic changes in the carpal bones preoperatively, and most changes were not evident one to two years after the operation. We speculate that the degenerative cystic changes of the lunate and triquetrum can resolve after adequate decompression by ulnar shortening and targeted arthroscopic treatment.

Some reports have suggested that oblique ulnar osteotomy has the advantage of more rapid healing compared with a transverse osteotomy ${ }^{14,15}$. However, others have found no difference in healing times ${ }^{16,17}$. Besides the type of osteotomy, other factors, such as cigarette smoking ${ }^{18}$ and the amount of heat generation with the microsagittal saw blade during ulnar osteotomy ${ }^{19}$, are known to adversely influence the healing time of an osteotomized ulna. In the present study, there were no delayed unions or nonunions with use of a conventional transverse osteotomy.

In conclusion, ulnar shortening osteotomy performed in patients with idiopathic ulnar impaction syndrome resulted in improvement in the wrist scores and reduction of the subluxation of the distal radioulnar joint when present.

Goo Hyun Baek, MD

Moon Sang Chung, MD

Young Ho Lee, MD

Hyun Sik Gong, MD

Sanglim Lee, MD

Hyung Ho Kim, MD

Department of Orthopaedic Surgery, Seoul National University College of Medicine, 28 Yongon-Dong, Chongno-Gu, Seoul 110-744, South Korea. E-mail address for G.H. Baek: ghbaek@snu.ac.kr

The authors did not receive grants or outside funding in support of their research or preparation of this manuscript. They did not receive payments or other benefits or a commitment or agreement to provide such benefits from a commercial entity. No commercial entity paid or directed, or agreed to pay or direct, any benefits to any research fund, foundation, educational institution, or other charitable or nonprofit organization with which the authors are affiliated or associated.

doi:10.2106/JBJS.D.02983

\section{References}

1. Friedman SL, Palmer AK. The ulnar impaction syndrome. Hand Clin. 1991; 7:295-310.

2. Coleman DA, Blair WF, Shurr D. Resection of the radial head for fracture of the radial head. Long-term follow-up of seventeen cases. J Bone Joint Surg Am. 1987;69:385-92.

3. Chun S, Palmer AK. The ulnar impaction syndrome: follow-up of ulnar shortening osteotomy. J Hand Surg [Am]. 1993;18:46-53.

4. Loh YC, Van Den Abbeele K, Stanley JK, Trail IA. The results of ulnar shortening for ulnar impaction syndrome. J Hand Surg [Br]. 1999;24:316-20.

5. Nakamura R, Horii E, Imaeda T, Tsunoda K, Nakao E. Distal radioulnar joint subluxation and dislocation diagnosed by standard roentgenography. Skeletal Radiol. 1995;24:91-4.

6. Insall J, Falvo KA, Wise DW. Chondromalacia patellae. A prospective study. J Bone Joint Surg Am. 1976;58:1-8.

7. Jung JM, Baek GH, Kim JH, Lee YH, Chung MS. Changes in ulnar variance in relation to forearm rotation and grip. J Bone Joint Surg Br. 2001;83: 1029-33.

8. Chen WS, Shih $\mathrm{CH}$. Ulnar variance and Kienbock's disease. An investigation in Taiwan. Clin Orthop Relat Res. 1990;255:124-7.

9. Nakamura R, Tanaka Y, Imaeda T, Miura T. The influence of age and sex on ulnar variance. J Hand Surg [Br]. 1991;16:84-8.

10. Schuind FA, Linscheid RL, An KN, Chao EY. A normal data base of postero- anterior roentgenographic measurements of the wrist. J Bone Joint Surg Am. 1992; 74:1418-29.

11. Kristensen $S S$, Thomassen $E$, Christensen $F$. Ulnar variance determination. J Hand Surg [Br]. 1986;11:255-7.

12. Nagle DJ. Arthroscopic treatment of degenerative tears of the triangular fibrocartilage. Hand Clin. 1994;10:615-24.

13. Deitch MA, Stern PJ. Ulnocarpal abutment. Treatment options. Hand Clin. 1998;14:251-63.

14. Rayhack JM, Gasser SI, Latta LL, Ouellette EA, Milne EL. Precision oblique osteotomy for shortening of the ulna. J Hand Surg [Am]. 1993;18:908-18.

15. Chen NC, Wolfe SW. Ulna shortening osteotomy using a compression device. J Hand Surg [Am]. 2003;28:88-93.

16. Fricker R, Pellegrino A, Pfeiffer KM, Troeger $H$. [Wrist impaction syndrome after distal radial or forearm fractures treated by ulnar osteotomy. Surgical techniques and results in 26 cases]. Ann Chir Main Memb Super. 1997;16:285-91. French.

17. Wehbe MA, Cautilli DA. Ulnar shortening using the $A O$ small distractor. J Hand Surg [Am]. 1995;20:959-64.

18. Chen F, Osterman AL, Mahony K. Smoking and bony union after ulna-shortening osteotomy. Am J Orthop. 2001;30:486-9.

19. Firoozbakhsh K, Moneim MS, Mikola E, Haltom S. Heat generation during ulnar osteotomy with microsagittal saw blades. lowa Orthop J. 2003;23:46-50. 